\title{
Immunohistochemical Evaluation of Type IV Collagen Alpha Chains in Oral Malignant Melanoma
}

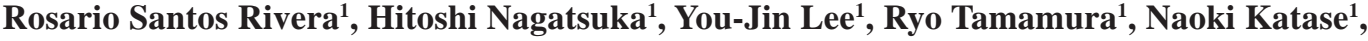 \\ Liu Yue-Heng ${ }^{2}$, and Noriyuki Nagai ${ }^{1}$
}

${ }^{1}$ Okayama University, Graduate School of Medicine, Dentistry and Pharmaceutical Sciences, ${ }^{2}$ Beijing University, Shenzhen Hospital, China

\section{Introduction}

The basement membrane (BM) is mainly composed of type IV collagen composed of triple combinations of 6 distinct a chains ( $\alpha 1-\alpha 6)$. Invasive and metastatic melanoma cells form cluster surrounded by BM-like structures. The objective of the study was to determine the immunohistochemical pattern of type IV collagen a chain at the BM-like structures surrounding melanoma clusters.

\section{Materials and methods}

Paraffin blocks of human tissues of 1 in situ OMM, 1 invasive OMM, 6 invasive with in situ OMM, 5 metastatic OMM to lymph nodes were sectioned. Type IV collagen $\alpha$ chain specific rat monoclonal antibodies (provided by Dr. Naito and Dr. Sado) were used according to their specific dilutions. AEC chromogen was used to reveal the antigenic sites.

\section{Results}

$\alpha 1,2,5$ and 6 were constantly detected at the BM of the oral epithelium (Fig.1). $\alpha 1,2,5$ and 6 were intermittently detected at the BM of the oral epithelium in in situ OMM (Fig.2). $\alpha 1$ and 2 were intermittently detected at the BM of the oral epithelium while $\alpha 5$ and 6 were negative in early invasive OMM (Fig.3). In invasive OMM, $\alpha 1$ and 2 were constantly detected at the BM-like structures surrounding nodular nests (Fig.4) and were intermittently detected at the BM-like structures surrounding sheet-like nests (Fig.5). The metastatic melanoma cells likewise formed clusters and $\alpha 1$ and 2 were constantly detected at the BM-like structures surrounding nodular nests (Fig.6) while intermittent in sheet-like nests.

\section{Discussion}

The gradual loss of type IV collagen $\alpha$ chains at the BM of the oral epithelium is associated with the progression of OMM. Type IV collagen $\alpha 1$ and 2 are more stable compared to $\alpha 5$ and 6. BMs are dynamic structures that are not only degraded but also deposited around melanoma cell clusters and the distribution pattern of type IV collagen a chains varies depending on the architecture of the nest. These suggest that type IV collagen a chains can be significant markers of oral melanocytic progression.
Fig.1

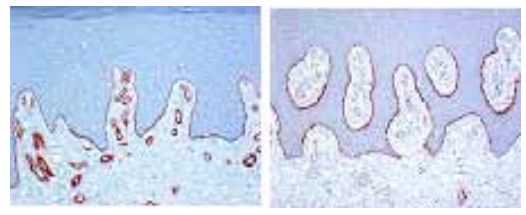

Fig. $1 \alpha 2$ and 5 were constantly detected at the $\mathrm{BM}$ of normal oral epithelium.

Fig.4

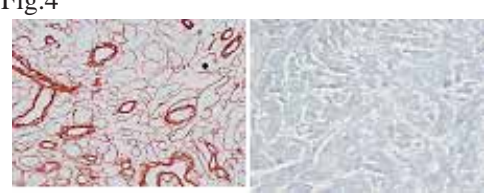

Fig.4 $\alpha 2$ was constantly detected around the nodular nests in invasive melanoma while $\alpha 5$ was negative.

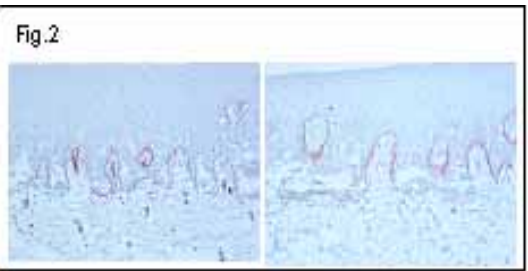

Fig. $2 \alpha 2$ and 5 were intermittently detected at the $\mathrm{BM}$ of the oral epithelium in in situ melanoma.

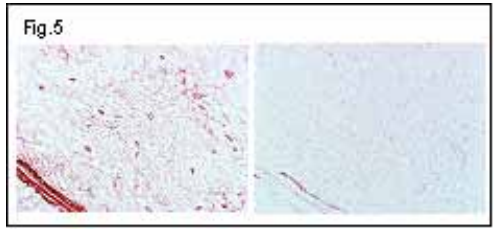

Fig. $5 \alpha 2$ was intermittently detected around sheet-like nests in invasive melanoma while $\alpha 6$ was negative.

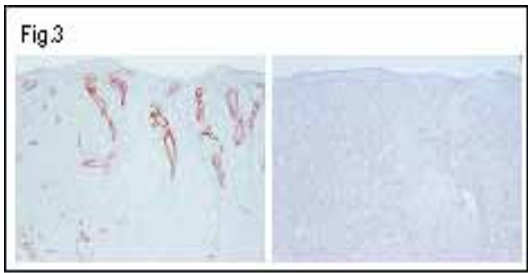

Fig.3 $\alpha 2$ was intermittently detected in early invasive melanoma while $\alpha 6$ was negative.

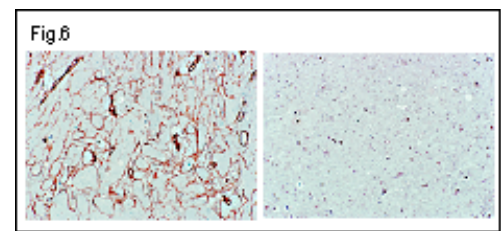

Fig. $5 \alpha 2$ was intermittently detected around sheet-like nests in invasive melanoma while $\alpha 6$ was negative.

Table. Summary of IHC of oral epithelium in different OMM and melanoma nests in invasive and metastatic OMM.

\begin{tabular}{lllll} 
Oral epithelium & $\alpha 1$ & $\alpha 2$ & $\alpha 5$ & $\alpha 6$ \\
\hline Normal & + & + & + & + \\
In situ OMM & \pm & \pm & \pm & \pm \\
Early invasive & \pm & \pm & - & - \\
\hline
\end{tabular}

\begin{tabular}{lcccc} 
Melanoma nest & $\alpha 1$ & $\alpha 2$ & $\alpha 5$ & $\alpha 6$ \\
\hline Nodular nest & + & + & - & - \\
Sheet-like nest & \pm & \pm & - & -
\end{tabular}

